\title{
Investigation of the Effect of Rotation Speed on Vibration Responses of Transmission System
}

\author{
Xiaogang Liu , Zhaoyu Wu, Jie Lu, and Jinli Xu \\ School of Mechanical and Electronic Engineering, Wuhan University of Technology, Hubei 430070, China
}

\begin{abstract}
In the operating process, it is found the vibration of main reducer reaches a maximum value when certain types of vehicles are running at a speed around 4000 RPM. However, how the rotation speed of engine affects the vibration responses to automobile transmission system has not been investigated theoretically in details. To investigate this problem, the transmission system of automobile is simplified to a drive-final shaft system in this research, and a coupled vibration model of drive-final shaft system is developed. This model is used to simulate the vibration response to transmission system at different rotation speeds. Simulation results show that the torsional vibration responses reach the maximum when the rotation speeds are 3800 RPM and 4200 RPM and the vibration responses of pinion reach the maximum value when the rotation speeds are 4000 RPM and 4200 RPM. Moreover, finite element analysis is conducted to investigate the reason for this phenomenon. It is found that the torsional vibration responses reach the maximum value when the excitation frequency of engine is close to the resonance frequency of drive shafts. This research provides an effective method to analyse the vibration characteristics of automobile transmission system.
\end{abstract}

\section{Introduction}

As the automobile transmission system generates vibration and noise in the process of power transmission, the analysis of vibration and noise is especially crucial for improving the quality of automobile. The vibration and noise mainly generated by the inhomogeneity of the transfer velocity of the universal joint, the arrangement of drive shaft and the coupled vibration of gear pair of main reducer [1-3]. The torque and rotation speed of engine transmit the power directly to wheels through the transmission system, so developing a reasonable automobile transmission system model is quite necessary.

Generally, the vibration of main reducer is mainly due to the meshing vibration of bevel gears, which had been investigated intensively. One seven degree-of-freedom dynamic model of bevel gear system was developed by defining the relative displacement along the line of action in [4], where backlash, time-varying mesh stiffness and transmission error were considered. A mathematical model of bevel gears was developed in [5], which adopted multi-body and multiple-degree-of-freedom dynamic modeling to characterise the mesh and dynamic behaviour and predict vibration response.

Engine torque is an important parameter that affects vibration of vehicle transmission system. It was suggested that fluctuation of engine torque is the main source of noise and vibration of transmission system [6]. Torsional vibration response can be reduced to a certain extent by installing the elastic connector in transmission system. One three degree-of-freedom dynamic model of engine was developed [7], which concluded that vibration amplitude increases due to engine excitation when the pulsating torque of engine increases; while the average torque of engine had little effect on jitter.

For the transmission system of automobile, it is necessary to get more factors involved in modeling. Lee et al. [8] developed a mathematical model of an automotive transmission, which involves the flexibility of the shafts, bearings, gear teeth and gyroscopic effects of geared rotors. From the study on force analysis of drive shaft [9], it was found that the plunging force and generated axial force were the most influential quantities related to the vibrational responses of drive shaft. The effect of engine speed on the torsional vibration of drive shaft was investigated [10] and the increase of torsional inertia led to a decrease in the frequency of torsional vibration.

Although different models for transmission system have been developed, there are still some problems have not been addressed. For instance, it is found that certain types of vehicles often vibrate dynamically when the engine is rotating around 4000 RPM, but no detailed illustration for this phenomenon has ever been provided. In this research, the effect of torsional vibration of drive shafts and coupled vibration of gears are integrated to investigate this problem. Based on the models developed previously [3, 11-13], a multi degree of freedom transmission system model is developed by integrating torsional vibration of drive shaft with coupled vibration of gear pair of main reducer. Using this model, the torsional vibrations of transmission system at various speeds are 
simulated, and the reason for this phenomenon is illustrated.

\section{Methodologies}

In this part, the contact forces of the gear pair of main reducer are analysed, and the coupled vibration model of gear pair is developed. On the other hand, the torsional vibration model of drive shaft is developed using lumped mass method. Finally, the model of transmission system can be developed by integrating these two models.

\subsection{Hypoid gear model}

As one important part of the drive bridge assembly, the main reducer is used to reduce rotation speed and increase torque. In the process of transmission, vibration and noise are generated due to the meshing of gears in main reducer.

For the convenience of analysis, the uniform force is substituted as a concentrated force on the middle point of the gear tooth. The forces on the pinion was provided in [3],

$$
\left\{\begin{array}{c}
F_{n}=k_{m}(t) f\left(x_{n}\right)+c_{m} \dot{x}_{n} \\
F_{x}=-F_{n} \cos \alpha_{n} \sin \beta_{1} \cos \delta_{1} \\
+F_{n} \sin \alpha_{n} \sin \delta_{1}=-a_{1} F_{n} \\
F_{y}=-F_{n} \cos \alpha_{n} \sin \beta_{1} \cos \delta_{1} \\
-F_{n} \sin \alpha_{n} \sin \delta_{1}=-a_{2} F_{n} \\
F_{z}=-F_{n} \cos \alpha_{n} \cos \beta_{1}=-a_{3} F_{n}
\end{array}\right.
$$

where $F_{n}$ is the normal dynamic meshing force of pinion, and $F_{x}, F_{y}$ and $F_{z}$ are the meshing force of pinion in $x, y$ and $z$ direction, $\delta_{1}$ is the pitch cone angle of pinion, $\beta_{1}$ is the average spiral angle, $\alpha_{n}$ is the average pressure angle, $c_{m}$ is the average damping of hypoid gear, $\dot{x}_{n}$ is the relative velocity between two gears at meshing point in the normal direction, $k_{m}(t)$ is the gear pair meshing stiffness, $f\left(x_{n}\right)$ is the backlash function, $x_{n}$ is the relative displacement between two gears at meshing point in the normal direction.

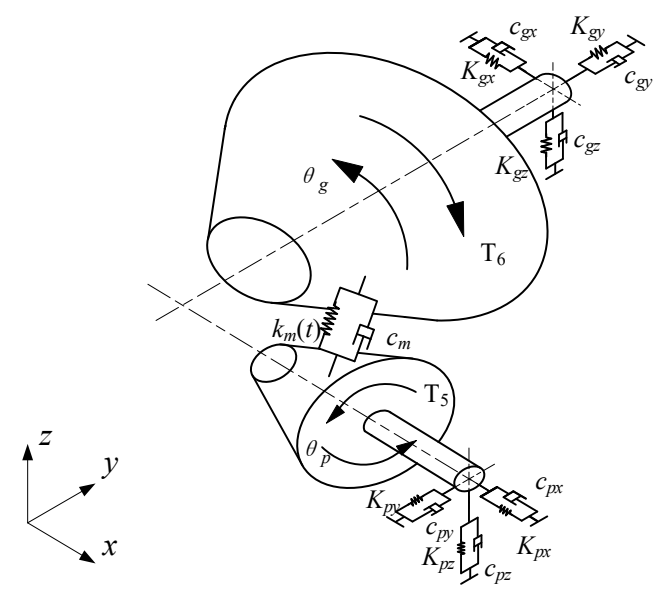

Fig. 1. Coupled vibration model of hypoid gears.

Due to the elastic deformation of bearings and shaft of pinion, displacements in three directions can be generated for the center of gear. In the model, pinion and gear are equivalent to lumped mass or rotary inertia. In this model, the shaft of pinion and gear are simplified as massless rigid body; the bearing is simplified as a massless spring and a damper; the gear pair is simplified as a spring and a damper, as presented in the Fig. 1 .

Using the lumped mass method, the gear coupled vibration model is developed, which can be expressed as,

$$
\left\{\begin{array}{c}
m_{p x} \ddot{x}_{p}+c_{p x} \dot{x}_{p}+K_{p x} x_{p}=F_{x} \\
m_{p y} \ddot{y}_{p}+c_{p y} \dot{y}_{p}+K_{p y} y_{p}=F_{y} \\
m_{p z} \ddot{z}_{p}+c_{p z} \dot{z}_{p}+K_{p z} z_{p}=F_{z} \\
J_{5} \ddot{\theta}_{P}-F_{z} R_{5}=T_{5} \\
m_{g x} \ddot{x}_{g}+c_{g x} \dot{x}_{g}+K_{g x} x_{g}=-F_{x} \\
m_{g y} \ddot{y}_{g}+c_{g y} \dot{y}_{g}+K_{g y} y_{g}=-F_{y} \\
m_{g z} \ddot{z}_{g}+c_{g z} \dot{z}_{g}+K_{g z} z_{g}=-F_{z} \\
J_{6} \ddot{\theta}_{g}+F_{z} R_{6}=-T_{6}
\end{array}\right.
$$

where $m_{p x}, m_{p y}$ and $m_{p z}$ are equivalent mass of pinion in three directions, which are equal in value; $m_{g x}, m_{g y}$ and $m_{g z}$ are equivalent mass of gear in three directions, which are equal in value; $c_{p x}, c_{p y}$ and $c_{p z}$ are equivalent damping of pinion in $x, y$ and $z$ direction; $c_{g x}, c_{g y}$ and $c_{g z}$ are equivalent damping of gear in $x, y$ and $z$ direction; $K_{p x}, K_{p y}$ and $K_{p z}$ are equivalent stiffness of pinion in $x$, $y$ and $z$ direction; $K_{g x}, K_{g y}$ and $K_{g z}$ the equivalent stiffness of gear in $x, y$ and $z$ direction; $x_{p}, y_{p}$ and $z_{p}$ are the displacement of pinion in $x, y$ and $z$ direction; $x_{g}, y_{g}$ and $z_{g}$ are the displacement of gear in $x, y$ and $z$ direction; $\theta_{p}$ and $\theta_{g}$ are the angular displacement of pinion and gear; $R_{5}$ and $R_{6}$ are radius of nodal circle of pinion and gear at meshing point.

\subsection{Nonlinear dynamic model of shaft-final drive system}

The vibration of the drive-final shaft system mainly derives from the external excitation of the transmission system and the internal excitation of the subsystem. The external excitations of the transmission system include the vibration produced by engine and the fluctuation of engine rotation speed. The internal excitations of the subsystem include the variation of load caused by the inhomogeneity of the velocity transfer of universal joint and the excitation caused by gear manufacturing error. The internal excitations and external excitations are considered when taking the drive shaft system and gear system as a whole system to model.

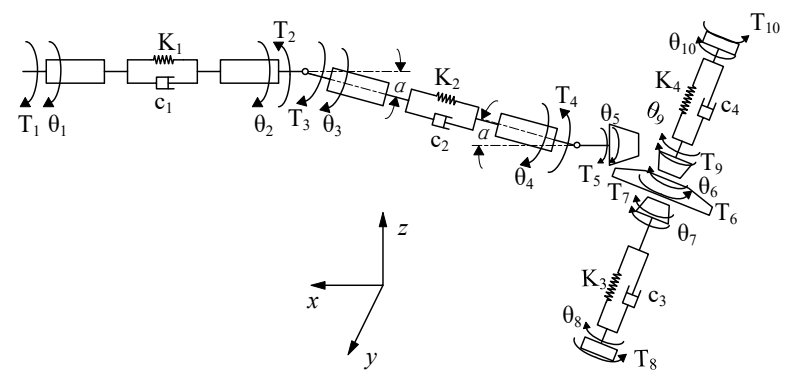

Fig. 2. Torsional vibration model of the shaft-final drive system where $K_{1}$ and $K_{2}$ are the equivalent torsional stiffness of the drive shafts; $K_{3}$ and $K_{4}$ are the equivalent torsional 
stiffness of left and right half drive shaft; $c_{1}$ and $c_{2}$ are the damping of the drive shafts; $c_{3}$ and $c_{4}$ are the damping of the left and right half drive shafts; $J_{1}, J_{2}, J_{3}$ and $J_{4}$ are the equivalent rotary inertia of the drive shafts; $J_{7}$ and $J_{8}$ are the equivalent rotary inertia of the left half drive shaft and the left hub around its axis.

The drive-final shaft system is a complex elastic system. To simplify analysis and calculation, some factors should be ignored when developing its dynamic model, and take it as a multi degree of freedom discrete analysis model. The model of drive-final shaft system has been developed by the lumped mass method, and the simplified model of the torsional vibration of drive-final shaft system is shown in Fig. 2,

Integrated with the model of gear pair and the angle relationship of drive shaft [13], the torsional vibration model of the drive-final shaft system can be expressed as Equation (3).

$$
\left\{\begin{array}{c}
J_{1} \ddot{\theta}_{1}+c_{1}\left(\dot{\theta}_{1}-\dot{\theta}_{2}\right)+K_{1}\left(\theta_{1}-\theta_{2}\right)-T_{1}=0 \\
J_{2} M_{2} \ddot{\theta}_{2}+J_{3}\left(\frac{\ddot{\theta}_{2}}{A-B \cos 2 \theta_{2}}-\frac{2 B \sin 2 \theta_{2}}{\left(A-B \cos 2 \theta_{2}\right)^{2}} \dot{\theta}_{2}{ }^{2}\right) \\
+c_{1} M_{2}\left(\dot{\theta}_{2}-\dot{\theta}_{1}\right)+c_{2}\left(\frac{\dot{\theta}_{2}}{A-B \cos \theta_{2}}-\frac{\dot{\theta}_{5}}{A-B \cos \theta_{5}}\right) \\
+K_{1} M_{2}\left(\theta_{2}-\theta_{1}\right)+ \\
K_{2}\left(\arctan \frac{\tan \theta_{2}}{\cos \alpha}-\arctan \frac{\tan \theta_{5}}{\cos \alpha}\right)=0 \\
J_{5} M_{5} \ddot{\theta}_{5}+J_{4}\left(\frac{\ddot{\theta}_{5}}{A-B \cos 2 \theta_{5}}-\frac{2 B \sin 2 \theta_{5}}{\left(A-B \cos 2 \theta_{5}\right)^{2}} \dot{\theta}_{5}{ }^{2}\right)+ \\
c_{2}\left(\frac{\dot{\theta}_{5}}{A-B \cos \theta_{5}}-\frac{\dot{\theta}_{2}}{A-B \cos \theta_{2}}\right)+ \\
K_{2}\left(\arctan \frac{\tan \theta_{5}}{\cos \alpha}-\arctan \frac{\tan \theta_{2}}{\cos \alpha}\right)-F_{z} R_{5} M_{5}=0 \\
2 J_{7} \ddot{\theta}_{6}+J_{6} \ddot{\theta}_{6}+2 c_{3}\left(\dot{\theta}_{6}-\dot{\theta}_{8}\right) \\
+2 K_{3}\left(\theta_{6}-\theta_{8}\right)+F_{z} R_{6}=0 \\
J_{8} \ddot{\theta}_{8}+c_{3}\left(\dot{\theta}_{8}-\dot{\theta}_{6}\right)+K_{3}\left(\theta_{8}-\theta_{6}\right)=-T_{8} \\
m_{p x} \ddot{x}_{p}+c_{p x} \dot{x}_{p}+K_{p x} x_{p}=F_{x} \\
m_{p y} \ddot{y}_{p}+c_{p y} \dot{y}_{p}+K_{p y} y_{p}=F_{y} \\
m_{p z} \ddot{z}_{p}+c_{p z} \dot{z}_{p}+K_{p z} z_{p}=F_{z} \\
m_{g x} \ddot{x}_{g}+c_{g x} \dot{x}_{g}+K_{g x} x_{g}=-F_{x} \\
m_{g y} \ddot{y}_{g}+c_{g y} \dot{y}_{g}+K_{g y} y_{g}=-F_{y} \\
m_{g z} \ddot{z}_{g}+c_{g z} \dot{z}_{g}+K_{g z} z_{g}=-F_{z}
\end{array}\right.
$$

\section{Results and discussion}

The amplitudes of angular displacement and angular vibration of transmission system are simulated and presented in Fig. 3.

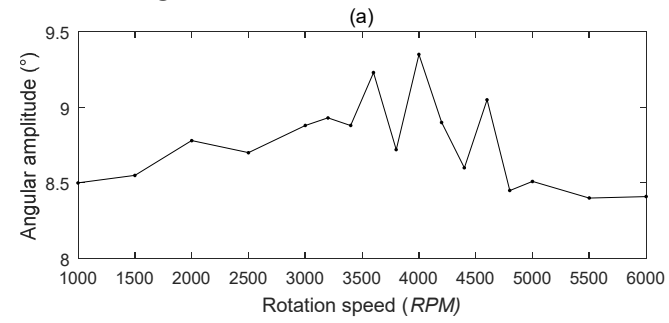

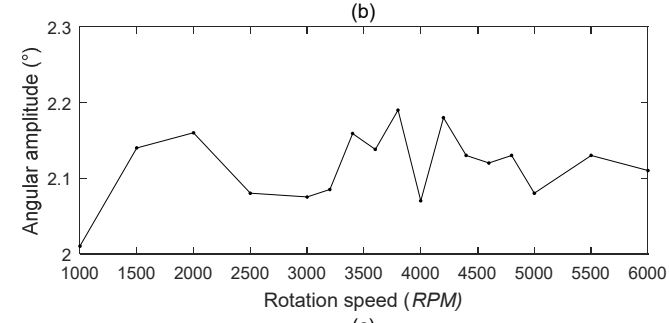

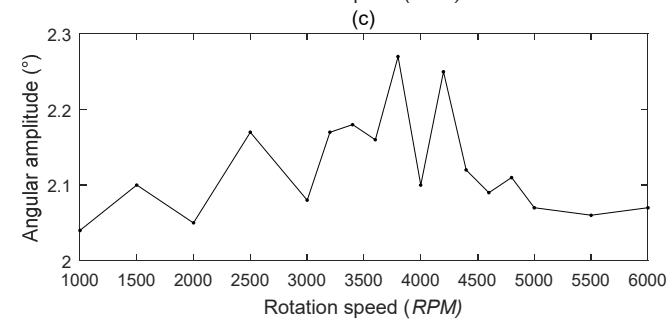

Fig. 3. Torsional vibration responses of transmission system at different rotation speeds at various parts: (a) drive shaft output terminal, (b) rear axle output terminal, (c) hub.

As can be seen from Fig. 3, the changing trend of angular amplitude of drive shaft output terminal, rear axle output terminal and hub are nearly the same at different rotation speeds. Taking torsional vibration response of drive shaft output terminal in Fig. 3 (a) as an example, angular displacement amplitude reaches maximum value when the speed is 3800 RPM and 4200 RPM.

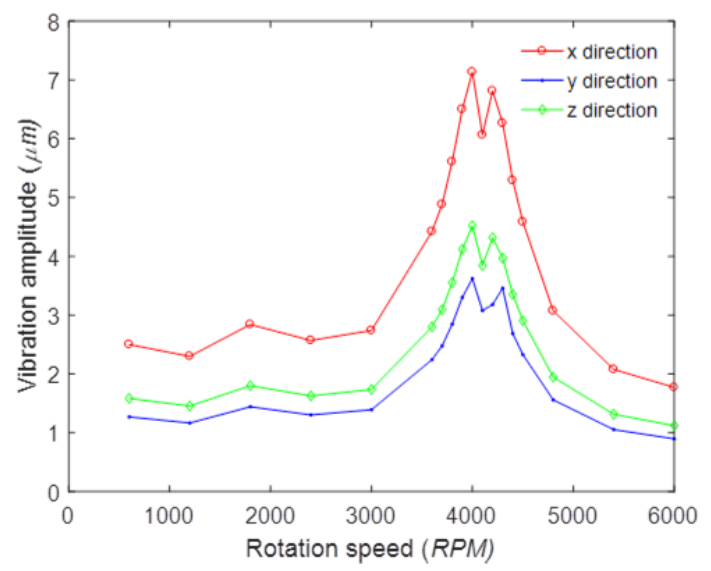

Fig. 4. Vibration amplitude of pinion at different rotation speeds. It can be seen from Fig. 4 that the trend in the change of vibration amplitudes of pinion in $x, y$ and $z$ directions are similar at different rotation speeds, only that the value of amplitudes are different. The general trend is that the amplitude increases before rotation speed reaches 4000 RPM, and decreases beyond 4200 RPM.

To explore the reasons for the two peaks, the vibration characteristics of drive shaft have been analysed by using finite element analysis, and first six mode frequencies are listed in Table 1.

Table 1. The first six order frequency of drive shaft

\begin{tabular}{ccccccc}
\hline Order & 1 & 2 & 3 & 4 & 5 & 6 \\
\hline Frequency $/ \mathrm{Hz}$ & 130.9 & 140.2 & 415.2 & 442.2 & 636.2 & 906.6 \\
\hline
\end{tabular}

The dominant frequency of drive shaft is $130.9 \mathrm{~Hz}$, whose mode shape is presented in Fig. 5 (a), which shows that drive shafts vibrate in $y$ direction. As the output torque is not uniform, when the rotation speed is 3800 RPM, the excitation frequency of drive shafts from engine is $126.7 \mathrm{~Hz}$, whose value is quite close to the dominant 
frequency of drive shafts. Therefore, resonance causes a dramatic torsional vibration response of drive shafts. Similarly, when the rotation speed is 4200 RPM, the excitation frequency of drive shafts is $140 \mathrm{~Hz}$, whose value is quite close to the second order resonance frequency of drive shafts, whose mode shape is presented in Fig. 5 (b), which show that the drive shafts vibrate in $y$ direction.

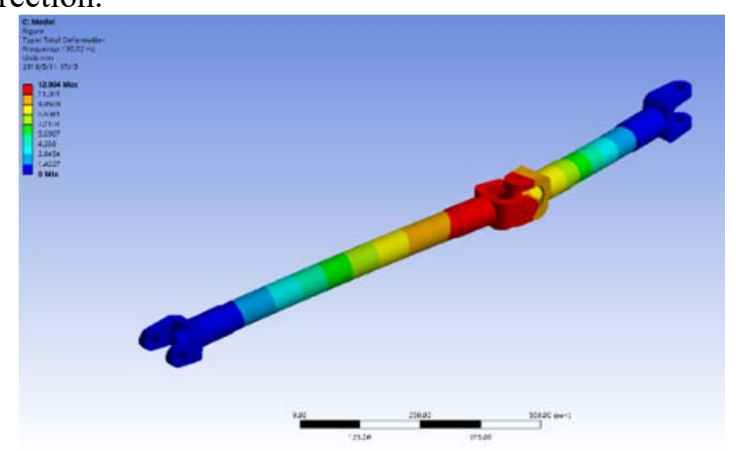

(a)

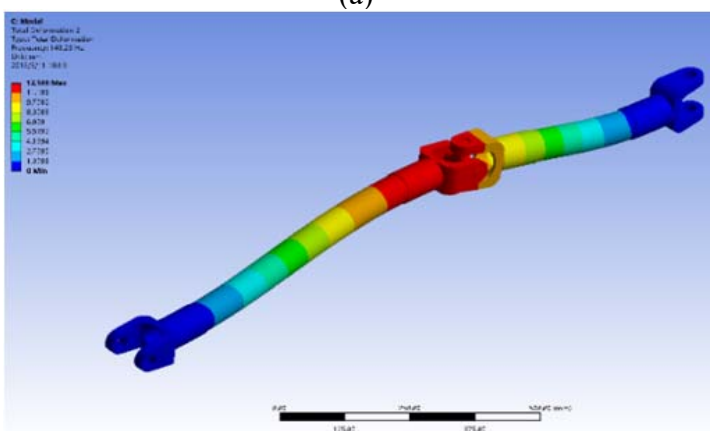

(b)

Fig. 5. Mode shapes of drive shafts: (a) the first order, (b) the second order.

\section{Conclusion}

A model integrating the torsional vibration and coupled vibration is developed in this research, with which the vibration responses of transmission system are acquired quantitatively rather than qualitatively. Using this model, the effect of rotation speed on transmission system is investigated. Simulation results show that torsional vibration amplitudes reach the maximum at the rotation speeds of 3800 RPM and 4200 RPM and the torsional vibration responses of drive shaft output terminal is the largest. In addition, the vibration amplitude of pinion in all directions increase continuously with rotation speed before it reaches 4000 RPM, and decrease when beyond 4200 RPM. To explore the reason for this phenomenon, finite element analysis is conducted, and the results show that the dramatic vibration at certain rotation speed is due to that the excitation frequency of drive shafts is close to its inherent frequency. Therefore, it seems that this model provides an effective method to analyse the vibration response of transmission system, and that this model can be applied further in other investigations about the vibration of transmission system.

The authors would like to acknowledge the funding from National Natural Science Foundation of China (Grant no.
51505352) and National Ministry of Industry and Information (Grant no. 2159999).

\section{References}

1. $\mathrm{Xu} \mathrm{J}$ et al. (2014) Research on Automobile Coupled Vibration between Transmission Shaft and Drive Axle Gears. Advanced Materials Research 915-916: 76-81.

2. $\mathrm{Xu} \mathrm{J}$ et al. (2015) Effects of the transmission shaft on the main reducer vibration based on ADAMS and experimental demonstration. Australian Journal of Mechanical Engineering.

3. Xu J Zeng F and $\mathrm{Su}$ X (2017) Coupled Bending-Torsional Nonlinear Vibration and Bifurcation Characteristics of Spiral Bevel Gear System. Shock and Vibration 1(2017): 1-14.

4. Yang X Zhou X and $\mathrm{Hu} \mathrm{H}$ et al. (2009) Nonlinear vibration characteristics of helical bevel gear systemand parameters influences. Journal of Zhejiang University 43(3): 505-510.

5. Peng T (2010) Coupled Multi-body Dynamic and Vibration Analysis of Hypoid and Bevel Geared Rotor System. Dissertations \& Theses - Gradworks.

6. Kim G W Shin S C (2015) Research on the torque transmissibility of the passive torsional vibration isolator in an automotive clutch damper. Proceedings of the Institution of Mechanical Engineers Part D Journal of Automobile Engineering 229(13).

7. Yang L K Li H Y Ahmadian M et al. (2015) Analysis of the influence of engine torque excitation on clutch judder. Journal of Vibration \& Control 14(3): 385-396.

8. Lee HW Park SH and Park MW et al. (2009) Vibrational Characteristics of Automotive transmission. International Journal of Automotive Technology 10(4): 459-467.

9. Sa J and Kang T (2013) A Study on the Characteristics of Vibration Due to the Forces of Drive Shaft. Journal of The Korean Society of Manufacturing Technology Engineers 22(4): 708-716.

10. Wang E Jing-Jing $X$ and Huang $Z$ et al. (2012) Analysis on Torsional Vibration for Drive Shafts of Engine Test Bed. Agricultural Equipment \& Vehicle Engineering.

11 Liu X and Meehan PA (2015) Wheel squeal noise: a simplified model to simulate the effect of rolling speed and angle of attack. Journal of Sound \& Vibration 338: 184-198.

12. Liu X and Meehan PA (2016) Investigation of Squeal Noise under Positive Friction Characteristics Condition Provided by Friction Modifiers. Journal of Sound \& Vibration 371: 393-405.

13. Xu J Su X and Bo P (2015) Numerical Analysis and Demonstration: Transmission Shaft Influence on Meshing Vibration in Driving And Driven Gears. Shock and Vibration 2015(10): 1-10. 